\title{
Titi Semantics: \\ Context and Meaning in Titi Monkey Call Sequences*
}

\author{
Philippe Schlenker ${ }^{\mathrm{a}}$, Emmanuel Chemla $^{\mathrm{b}}$, Cristiane Cäsar ${ }^{\mathrm{c}}$, Robin Ryder $^{\mathrm{d}}$, Klaus Zuberbühler $^{\mathrm{e}}$
}

Revised, February 8, 2016.

To appear in Natural Language and Linguistic Theory.

\begin{abstract}
Cäsar et al. 2013 show that the structure of Titi monkey call sequences can, with just two call types (A and B), reflect information about predator type and predator location. Using the general methods of Schlenker et al. 2014, to appear, a, b, we ask what these observations show about the 'linguistic' structure of Titi calls. We first demonstrate that the simplest behavioral assumptions make it challenging to provide lexical specifications for A- and B-calls: B-calls rather clearly have the distribution of highly underspecified calls; but A-calls are also found in highly heterogeneous contexts (e.g. they are triggered by 'cat in the canopy' and 'raptor on the ground' situations). We discuss two possible solutions to the problem. One posits that entire sequences are endowed with meanings that are not compositionally derived from their individual parts (a related idea was proposed by Arnold and Zuberbühler to analyze pyow-hack sequences in Putty-nosed monkeys). The second solution, which we consider to be superior, takes sequences to have no structure besides concatenation: the Bcall is a general call, the A-call is used for serious non-ground threats, and each call reflects information about the environment at the time at which it is uttered. The composition of Cäsar et al.'s sequences is seen to follow from the interaction between call meaning, rules of competition among calls, and more sophisticated assumptions about the environmental context. In the end, a detailed analysis of the division of labor between semantics, pragmatics and the environmental context yields a simple and explanatory analysis of sequences that initially seemed to display a complex mapping between syntax and semantics.
\end{abstract}

\footnotetext{
We are very grateful to three anonymous referees and to Editor Ad Neeleman for very helpful comments and Grant acknowledgments:

Cäsar: The research leading to these results received funding from the CAPES-Brazil, FAPEMIG-Brazil, S.B. LEAKEY TRUST and the University of St Andrews.

Chemla and Schlenker: Research by Schlenker and Chemla was conducted at Institut d'Etudes Cognitives, Ecole Normale Supérieure - PSL Research University. Institut d'Etudes Cognitives is supported by grants ANR-10-LABX-0087 IEC et ANR-10-IDEX-0001-02 PSL.

Schlenker: The research leading to these results received funding from the European Research Coucil under the European Union's Seventh Framework Programme (FP/2007-2013) / ERC Grant Agreement n³24115FRONTSEM (PI:Schlenker).

Zuberbühler: The research leading to these results received funding from the European Research Council under ERC grant 'Prilang 283871' and also from the Swiss National Science Fondation under grant 'FN 310030_143359/1'.

${ }^{a}$ Institut Jean-Nicod (ENS - EHESS - CNRS), Département d'Etudes Cognitives, Ecole Normale Supérieure, Paris, France ; PSL Research University ; New York University, New York.

${ }^{\mathrm{b}}$ LSCP (ENS - EHESS - CNRS), Département d'Etudes Cognitives, Ecole Normale Supérieure, Paris, France ; PSL Research University.

${ }^{c}$ School of Psychology and Neuroscience, University of St Andrews, St Mary's Quad, St Andrews, Fife, United Kingdom ; Instituto de Ciências da Natureza, Universidade Federal de Alfenas, Alfenas, Brazil.

${ }^{\mathrm{d}}$ Centre de Recherche en Mathématiques de la Décision, Université Paris-Dauphine, Paris, France.

${ }^{\mathrm{e}}$ Centre for Cognitive Science, University of Neuchâtel, Neuchâtel, Switzerland ; School of Psychology \& Neuroscience, University of St Andrews, St Mary’s Quad, St Andrews, Fife, United Kingdom.
} 


\section{Introduction}

\subsection{The study of primate call meaning}

In the last 40 years, there has been increasing interest in the semantic content of alarm calls in several primates (see Seyfarth et al. 1980a, b for some pioneering work on vervet monkeys, and Zuberbühler 2009 for a survey), and rich data have been collected in naturalistic observations as well as in field experiments. Recently, Cäsar et al. 2013 showed that the call sequences of black-fronted Titi monkeys (Callicebus nigrifrons, South America) can, with just two call types (A and B), reflect information about predator type and predator location. Cäsar et al. 2013 concluded that black-fronted Titi monkeys have "complex, structurally organized sequences that have the potential to convey information about both location and type of a predator". Should one conclude that this call system displays a complex syntax/semantics interface? We re-visit these data using the general tools of contemporary semantics, and reach a negative conclusion: Cäsar et al.'s stereotyped sequences are better explained by an analysis in which sequences have no structure besides the concatenation of individual calls, each contributing its information independently from the others. But this conclusion will be reached on the basis of a detailed analysis of the 'division of labor' between call meaning, rules of competition among calls, and non-trivial properties of the environmental context. In particular, we will see that an initial attempt to specify meanings for elementary calls reaches a dead end, and that assumptions about the environmental context are crucial to obtain an appropriate analysis. We will also argue that the B-call is used as a very general (underspecified) call, while the A-call is used for serious non-ground threats, and that rules of competition are needed to explain why the B-call fails to be used in all sorts of situations - hence a mechanism of meaning enrichment comparable to 'scalar implicatures' in human language.

To situate our enterprise, we should start with three questions that have been the object of particular attention in recent debates on alarm calls:

(i) Call meaning: What is the information conveyed by elementary calls?

(ii) Call combination: Do some calls or sequences of calls have internal structure, and if so how is the meaning of the whole derived from the meaning of its parts?

(iii) Call competition: Are there informativity-based rules of competition among calls, mandating that more specific ones outcompete less specific ones when applicable?

Concerning call meaning (= (i)), an important distinction is whether calls directly encode information about a predator type, or information about properties of the threat (level, directional origin, etc), or a combination of both. In the first case (information about predator types), ethologists often say that the calls are 'functionally referential'. Summarizing recent research on animal communication, Cäsar et al. 2012 write:

"... it is not clear whether primates intend to produce calls that refer to specific external events, or whether they 
merely respond to "evolutionarily important" events that place them into different motivations. One way to address this has been by investigating whether associated variables, such as the level of threat experienced by the caller, can explain the caller's behaviour better than the predatory category (e.g., California ground squirrels: Owings and Virginia 1978). In some other species, it has been argued that alarm calls refer to both the level and type of threat (Manser et al. 2002; Templeton et al. 2005; Sieving et al. 2010). Chickadees (Poecile atricapilla), for instance, produce "seet" alarm call in response to flying raptors and a "chick-a-dee" alarm call in response to perched or stationary raptors, but their calls also provide information about the threat level (Templeton et al. 2005). Within the primate lineage, the predator type appears to have an overriding influence on alarm calling behaviour, with little evidence that variation in distance or direction has a major impact (e.g., vervet monkeys: Cheney and Seyfarth 1990, Diana monkeys: Zuberbühler 2000b)."

As we will see, our analysis of Titi monkey calls will crucially assign to the A-call a non-referential meaning, pertaining both to the position of the threat and to the level of danger.

Concerning call combination (= (ii)), two cases have received some attention in the primate lineage, pertaining to complex calls in Campbell's monkeys and to distinguished 'pyow-hack' sequences in Putty-nosed monkeys. In Campbell's monkeys (Cercopithecus campbelli campbelli, Africa), Ouattara et al. 2009 and Schlenker et al. 2014 argued that two roots, krak and hok, can be combined with the suffix -oo, which modifies their meaning in a regular fashion. For instance, hok is specified for serious non-ground threats, while hok-oo is used for weaker non-ground threats. At the syntactic level, Arnold and Zuberbühler 2006b showed that Putty-nosed monkeys (Cercopithecus nictitans martini, Africa) sometimes produce distinct pyow-hack sequences made of a small number of pyows followed by a small number of hacks; and these were shown both in quantitative observational data and in field experiments to be predictive of group movement. Importantly, however, they argued that pyow-hack sequences were syntactically combinatorial but not semantically compositional $^{1}$ because the meaning of the whole could not be derived from the meaning of its parts. For this reason they likened pyow-hack sequences to idioms in human language; this 'noncompositional' analysis was partly challenged by Collier et al. 2014 and Schlenker et al., to appear a. ${ }^{2}$ As we will see below, although Cäsar et al.'s rich Titi sequences initially seem to argue for a noncompositional analysis as well, an alternative in which each individual call has a constant meaning will turn out to be superior.

Concerning call competition (= (iii)), a common observation in primate call systems is that one call is used with a very general meaning (see for instance Wheeler and Fischer 2012 for a survey). ${ }^{3}$ For instance, in Campbell's monkeys krakoo is triggered in all types of contexts; and in

\footnotetext{
${ }^{1}$ A combination of expressions is (weakly) compositional if its meaning is a function of the meaning of its elementary parts and the way they are put together. See for instance Heim and Kratzer 1998 for a textbook introduction to a compositional analysis of meaning in human language.

${ }^{2}$ In addition, Schlenker et al. to appear $\mathrm{b}$ argue that Black-and-White Colobus sequences might involve complex calls. In a nutshell, their argument is that snort-roar sequences have a broader distribution than either of their component parts, which makes it difficult for the meaning of the whole to be obtained by conjoining the meanings of the parts. While they do not claim to decide the issue, they leave open the possibility that in snortroar sequences snort and roar should be likened to phonemes - in which case snort-roar sequences would be phonologically (but not necessarily morphologically or syntactically) complex.

${ }^{3}$ Besides noting that some calls are more general than others, Wheeler and Fischer propose an interesting generalization: "across species it tends to be the call associated with terrestrial predators that is given in other contexts, whereas the call associated with aerial predators tends to be context-specific and meet the criteria of
} 
Putty-nosed monkeys, pyow arises in very diverse contexts as well. In such cases, it is important to explain why the general call fails to appear (at least on its own) in contexts in which it could be used but some more specific calls are available. Schlenker et al. 2014, to appear a, b posit a mechanism by which a more specific call outcompetes a less specific one in case both are licensed by the external situation. In effect, this gives rise to a mechanism of 'meaning enrichment' akin to scalar implicatures in human language, although Schlenker et al. 2014, to appear, a, b emphasize that their version of the mechanism need not rely on rules of 'cooperative communication' or on a theory of mind (for a survey of scalar implicatures in different semantic traditions, see for instance Levinson 2000, and Schlenker, to appear, as well as the seminal ideas in Grice 1975 and Horn 1972). We will see that a similar mechanism is needed to analyze Titi monkey calls, but we will be equally cautious in not necessarily inferring that Titi monkeys have complex mind-reading abilities.

Finally, it should be noted that the standard assumption in the field is that, as a first approximation, most of the form and meaning of monkey calls is innately specified because unrelated monkeys use similar calls in comparable ways; ${ }^{4}$ but given how little is known in terms of Titi call acquisition, we won't have anything to say on this topic in the present study.

\subsection{The interest of Titi 'quiet' calls}

In this study, we will focus on the 'quiet calls' of Titi monkeys, which present an important theoretical challenge (Cäsar et al. 2013). As mentioned, A- and B-calls organized in different sequences appear to encode information about both predator type and predator location. It might initially seem that these sequences display a complex syntax/semantics interface - a momentous potential finding that ought to be subjected to detailed linguistic analysis. We do so using the general methods of contemporary semantics, following Schlenker et al. 2014, to appear, a, b.

We demonstrate that the simplest behavioral assumptions make it difficult to provide lexical specifications for A- and B-calls: B-calls rather clearly have the distribution of general, underspecified calls; but A-calls are also found in highly heterogeneous contexts (e.g. they are triggered by 'cat in the canopy' and 'raptor on the ground' situations). We discuss two possible solutions to this challenge. One posits that entire sequences are endowed with meanings that are not compositionally derived from their individual parts, as proposed by Arnold and Zuberbühler in their analysis of pyow-hack sequences' in Putty-nosed monkeys (2006, 2008, 2012, 2013). The second solution combines a very simple analysis of individual calls with some more sophisticated assumptions about the environmental context. Specifically, we argue that the B-call is a general call, that the A-call is used for serious non-ground threats (hence uniformly leads to reactions of 'looking up'), and that sequences are just the concatenation of individual calls, each reflecting information

functional reference" (Wheeler and Fischer 2012 p. 200). This generalization holds true in our preferred analysis of Titi calls, as it did in the final analysis of Campbell's calls in Schlenker et al. 201.

${ }^{4}$ But see for instance Schlenker et al. 2014, footnote 8, for a list of cases in which monkey call realization or use is subject to apparent 'dialectal' variation. 
about the environment at the time of its utterance. It is the interaction between these simple lexical meanings and some non-trivial properties of the environment that turns out to give rise to Cäsar et al.'s rich sequences. Furthermore, as in Schlenker et al. 2014, to appear, a, b, rules of competition among calls are needed to explain why the general B-call fails to be used in contexts in which more specific calls are applicable. Overall, our final conclusions will be decidedly deflationary: Titi monkey sequences do not provide evidence for a complex syntax or syntax/semantics interface, although they do provide evidence for particularly subtle division of labor between call meaning, call competition, and properties of the environmental context.

\subsection{The import of semantic methods}

While monkey calls are not a standard object of study within linguistics, we believe that a detailed semantic study of Titi calls is justified on two grounds. First, the sophisticated sequences uncovered by Cäsar et al. 2013 are in need of explanation, and the possibility that they display a complex syntax/semantics interface must be confirmed or refuted from within linguistics. Second, the formal methods we will apply - pertaining in particular to the division of labor between semantics, pragmatics, and 'world' knowledge/context change - are standard in semantics but highly uncommon in ethology; semantic skills were thus crucial to the present endeavor. ${ }^{5}$ We emphasize, however, that we do not claim that Titi sequences have any non-trivial properties in common with human language. The only possible exception pertains to rules of competition among calls: in our final analysis, these are formally similar to 'scalar implicatures' in human language (although we certainly do not claim that they share an evolutionary origin with them). Importantly, when we use linguistic terminology (e.g. sentence, lexical specifications, etc.), we do so without implying that these formal objects have the same kind of properties in Titi calls and in human languages.

\subsection{Organization}

The rest of this article is organized as follows. We lay out the main generalizations about Titi sequences in Section 2. Our initial assumptions about modules of monkey meaning are outlined in Section 3. In Section 4, we explain why Titi sequences pose a theoretical challenge: B-calls rather clearly have the distribution of general, underspecified calls; but A-calls are also found in highly heterogeneous contexts, hence a difficulty in finding appropriate lexical specifications for them. In Section 5, we compare two main theories. The first one, a non-compositional analysis, assigns meanings to entire sequences, as was done by Arnold and Zuberbühler for pyow-hack sequences in

\footnotetext{
${ }^{5}$ We worked as follows. Primatologists on the team of authors provided the data. Linguists analyzed them and sought to find an analysis, but they were initially puzzled by the great difficulties they had in finding lexical specifications for individual calls (a problem that was not noted in Cäsar et al. 2013). After realizing that the difficulty was quite fundamental, as explained in Section 4, they explored an analysis with a less direct connection between call use and call context, thanks to the environmental assumptions discussed in Section 5.2. It was when the primatologists on the team confirmed that there was independent evidence for these assumptions that the final analysis was adopted.
} 
Putty-nosed monkeys. The second theory posits instead that calls are interpreted individually, and that sequences do not have syntactic structure besides call concatenation; but assumptions about the environment as well as rules of call competition are crucial to address the initial problem. ${ }^{6}$

\section{Titi Sequences}

In Cäsar et al.'s field experiments (Cäsar et al.'s 2013), cat and raptor models were place on the ground or in the canopy, and the ensuing vocalizations were recorded and analyzed. Remarkably, the two factors predator $=\{$ cat; raptor $\}$ and location $=\{$ on the ground; in the canopy $\}$ gave rise to four types of sequences obtained with just two quiet calls, the A-call and the B-call, as represented in slightly simplified form in (1) (note that Titis have other quiet and loud calls, as described in Cäsar 2011). In each case, our generalizations are based on the first 30 calls of the sequences triggered by the various situations; sample sequences are included in the Appendix. The restriction to 30 calls is motivated by the fact that, after that point, the patterns obtained with A and B calls just repeat the end of the 30-call sequence (although in response to terrestrial predators, Titis might after a while produce some different, louder calls). When average lengths are given, they are thus computed on the basis of these truncated sequences as well, hence with a maximum length of 30 for any given sequence.

(Notation: if $\mathrm{X}$ is any call, $\mathrm{X}^{+}$represents a series of at least one $\mathrm{X}$-call, and $\mathrm{X}^{++}$a series of at least two $\mathrm{X}$-calls. We write $\underline{\mathrm{X}}^{+}$and $\underline{\mathrm{X}^{++}}$for series that display these patterns with up to 3 extraneous calls interspersed.)

(1) Model predator experiments

a. Raptor in the canopy:

$\mathrm{A}^{++}$(4/5 sequences; the 5 th contains interspersed O-calls; average length of the 5 sequences $=26.8$ calls)

b. Raptor on the ground: $\mathrm{A}^{++} \mathrm{B}^{++}\left(5 / 7\right.$ sequences; $2 / 7$ have the form $\left.\mathrm{A}^{+}\right)$

c. Cat in the canopy: $\mathrm{A} \mathrm{B}^{++}$(4/6 sequences; $1 / 6$ has the form $\mathrm{X} \mathrm{A} \mathrm{B}^{++}$with an unidentified call $\mathrm{X})$

d. Cat on the ground: $\mathrm{B}^{++}(5 / 5$ sequences $)$

Importantly, Cäsar collected naturalistic data, which provide further useful information and is summarized in simplified form in (2); we note in particular that $\mathrm{B}^{++}$sequences occur in non-animalrelated contexts, as shown in (2)d.

(2) Naturalistic observations
a. Flying raptor:
$\mathrm{A}^{+} \quad(19 / 20$ sequences; average length of the 20 sequences $=$
b. Calling or perched raptor: 2.2 calls)
c. Capuchin in tree:
$\underline{\mathrm{A}^{++}} \quad(9 / 9 ;$ average length of the 9 sequences $=15.8$ calls $]$
d. Non-animal-related
(foraging/descending/feeding):
Diverse: $\mathrm{A}^{++}, \mathrm{A}^{++} \mathrm{B}^{++} \mathrm{A}^{++}$, etc with $\mathrm{C}$-calls interspersed (see Appendix)
$\mathrm{B}^{++} \quad(13 / 16)$

\footnotetext{
${ }^{6}$ Since our investigations are focused on the meaning of Titi sequences, we do not make reference in the present piece of the methods used by Kershenbaum et al. 2014, 2015 to study the syntax of animal sequences.

${ }^{7}$ Importantly, in most cases Cäsar could confirm that the calls were produced by only one individual, especially the first 30 calls under discussion here. For details, see Cäsar et al. 2013, Table 1, and pp. 2-3 of that paper's online supplementary materials.
} 
Simple inspection shows that the stereotyped call sequences in (1) encode information about both predator threat and predator location. Should we conclude that Titi monkeys can associate a complex syntax to complex meanings? We will argue that this is unlikely, and that the generalizations in (1) and (2) are compatible with an analysis in which each call has a simple meaning that pertains to the precise moment at which it is uttered; on this view, the complexity of the call sequences is due at least as much to the complexity of the environmental context - and the fact that it changes as calls are uttered - as to the Titi 'linguistic' system per se.

\section{Monkey Meanings: semantics, pragmatics and world knowledge}

Schlenker et al. 2014 (followed by Schlenker to appear, a, b) attempt to apply the general methods of formal linguistics to two apparent 'dialects' of male Campbell's monkey alarm calls. While they do not claim that there is any (non-trivial) formal resemblance between Campbell's and human semantics,

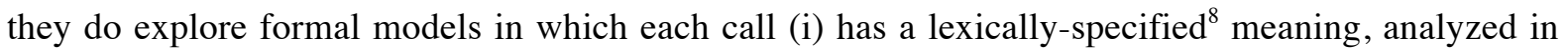
terms of applicability or 'truth' conditions; this meaning (ii) can be enriched by rules of competition among calls; and also (iii) modulated by world knowledge. In effect, they make use of three basic components of meaning that are standardly posited in analyses of human languages: literal meaning (= semantics); scalar implicatures, derived by assuming that under certain conditions the most informative sentence compatible with a situation is used (= a part of pragmatics); and world knowledge, which allows speakers to draw inferences that go beyond the information contributed by semantics and pragmatics alone.

Let us give a simple example to illustrate each module. For clarity we start from well-worn linguistic examples, but they serve to illustrate general methods that can be applied to various formal systems with truth or applicability conditions. It is uncontroversial that a speaker's mental dictionary must provide for a meaning difference between or and and; these are usually given the truth conditions in (3).

(3) Literal Meaning

a. $S$ and $S^{\prime}$ is true if and only if $S$ is true and $S^{\prime}$ is true

b. $S$ or $S^{\prime}$ is true if and only if $S$ is true, or $S^{\prime}$ is true, or both ('inclusive $o r^{\prime}$ )

Clearly, sentences of the form $S$ and $S^{\prime}$ and $S$ or $S^{\prime}$ give rise to all sorts of inferences which do not just depend on the meaning of $S, S^{\prime}$ and and/or, but also on what speakers know about the world. Two simple examples are given in (4).

(4) World Knowledge

a. John has been to Paris and he has been to Rome

$=>$ John hasn't been to Paris and Rome at the same time

b. John is in Paris or he is in Rome

$=>$ John isn't both in Paris and in Rome

\footnotetext{
${ }^{8}$ The term 'lexicon' pertains to the smallest units that carry information, with no implication that monkey calls are similar to human words.
} 
- While an utterance of (4)a gives rise to the inference that John has been to Paris and to Rome but not at the same time, there is certainly no need to add a negative temporal component to the lexical entry of and in (3)a to account for this observation: world knowledge guarantees that John couldn't have been in two places at the same time, and thus this inference need not be attributed to the semantics.

- The exclusive inference in (4)b can be treated in the same way. We might initially think that the not both inference argues for a different lexical entry for disjunction, one in which or both in (3)b is replaced with but not both ('exclusive or'). But a moment of thought suggests that here too world knowledge could just as well conspire with the lexical entry in (3)b to yield the observed inference, as John could not in Paris and Rome at the same time.

Other cases have been taken to argue for a third module, however, one which is distinct both from literal meaning and from world knowledge. A case in point is given in (5).

(5) Pragmatic Strengthening [here: Scalar Implicatures]

I'll invite John or Mary

$=>$ ? I'll invite John or Mary but not both

The exclusive inference in (5) initially looks like the one we had in (4)b, but upon further reflection it cannot be derived from world knowledge: in most situations, there is no reason whatsoever to think that the speaker couldn't both invite John and Mary. One might take this inference to argue for a revision of the lexical entry in (3)b, one in which exclusive or replaces inclusive or. But this option has been disfavored in recent linguistic theorizing, among others because it fails to account for the fact that it is by no means contradictory to say: I'll invite John or Mary - in fact, I'll invite them both. Without going into the details of a sophisticated line of argument, suffice it to say that contemporary theories are almost always based on the idea that the inference is due to competition between or and and: the sentence I'll invite John or Mary is compared to the alternative obtained by replacing or with and. The sentence I'll invite John and Mary is strictly more informative than the sentence I'll invite John or Mary. Hence one infers that the reason the speaker didn't choose the more informative version was that it was likely false. The result of this procedure is outlined in (6) (see Horn 1972 for some seminal ideas, and Schlenker, to appear for a recent survey).

(6) a. A sentence $S$ containing or is compared with alternatives $S$ ' obtained by replacing one or several occurrences of $o r$ with and.

b. If a sentence $S$ is uttered and an alterantive $S^{\prime}$ to $S$ is strictly more informative than $S$, one can usually infer that $S^{\prime}$ is false.

Slightly simplifying, Schlenker et al. 2014 (summarized in Schlenker, to appear b) make use of these three modules to analyze Campbell's alarm calls from the Tai forest, where the main predators are leopards and eagles: ${ }^{9}$

Semantics: They provide simple lexical specifications on which krak is a general call, hok is a nonground alarm call, and krakoo is a weak alarm call.

\footnotetext{
${ }^{9}$ For simplicity, we disregard hokoo from the present discussion; see Schlenker et al. 2014 for details.
} 
Pragmatics: They posit a rule of competition between krak, krakoo and hok, whose effect is that an utterance of krak usually (but not invariably) gives rise to the inference that hok and krakoo could not have been used. In the end, this yields the inference that there was a serious ground alarm: 'serious' because otherwise krakoo would have been used; and 'ground' because otherwise hok would have been used. Schlenker et al. 2014 do compare such rules of competition to scalar implicature in human language, but they are careful to add that such competition mechanisms do not entail that monkeys have a 'theory of mind': the rule by which a more informative call is to be preferred to a less informative one could be an automatic property of call use rather than the result of rules of cooperative information exchange ${ }^{10}$ (we will for the most part adopt this deflationary line of analysis in our own analysis of the relation between Titi calls and Titi cognition; we come back to this point in the conclusion).

World knowledge: Finally, they posit that the main serious ground alarm in the Tai forest is due to leopards, hence the impression that krak is a leopard alarm call. They argue that their analysis has the advantage of explaining why krak does have a few aerial uses in the Tai forest; and more importantly, why on Tiwai island, where eagles but not leopards are present, $k r a k$ is used as a general call, with its semantic meaning and without pragmatic strengthening. ${ }^{11}$

Context change: Importantly, the preferred analysis developed in Schlenker et al. 2014 has to rely not just on world knowledge but also on context change to provide an analysis of complex sequences. In brief, they have to explain why one and the same sequence could contain one or several hoks, as well as krakoo's. Given the 'weak alert' meaning of the latter, these sequences should come out as near-contradictions. The authors' solution is to relativize the meaning of each call to the precise time at which it is uttered, thus circumventing the problem of contradictions - with the result that the subjective seriousness of an alarm could change within the course of the utterance of a single sentence. While this is a rather theory-internal measure in Campbell's semantics, it will prove crucial in our analysis of Titi sequences.

As before, we should emphasize that the existence of a division between semantics, pragmatics and world knowledge/context change in monkey calls does not imply that the details of each module share non-trivial properties with human language. In fact, Schlenker et al. 2014 make no

\footnotetext{
${ }^{10}$ As they write, "in their simplest version scalar implicatures only require that subjects have at their disposal (i) a notion of satisfaction (to determine whether a sentence $S-$ e.g. $p$ or $q-$ is compatible with the situation at hand); (ii) a notion of scalar alternatives (to determine whether the sentence S' (e.g. $p$ and $q$ ) competes with the sentence S); and (iii) a notion of entailment (to determine whether $S$ ' is more informative than $S$ ). These three ingredients could suffice to yield the inference that if $p$ or $q$ was uttered, the more informative statement $p$ and $q$ is false.".

${ }^{11}$ World knowledge plays a crucial role to determine whether pragmatic strengthening should be applied or not. In a nutshell, Schlenker et al. 2014 posit that the reason pragmatic strengthening fails to be applied on Tiwai island is that it would yield a strengthened meaning which is almost never applicable, because there are no ground predators in that environment.
} 
such claim, with the limited exception of rules of competition among calls. And even there, they are cautious to state that monkey call competition certainly need not follow from Gricean principles of cooperative communication: a more informative call might outcompete a less informative one by way of a 'hardwired' procedure that does not appeal to speaker's intentions nor cooperativity.

\section{The Titi Challenge: Lexical Specifications}

The Titi generalizations in (1) and (2) pose a challenge for a semantic analysis based on simple (we'll soon say: simplistic) assumptions about the environment. In a nutshell, the problem is that the B-call occurs in contexts that are so diverse that it seems to be a general call, with a very weak lexical specification (note in particular that in (2)d above the B-call is used foraging/descending/feeding situations, which do not involve predators). But it turns out that within predator contexts the A-call also occurs in environments that fail to form a natural class, and hence it seems to function as a general predator alarm call. But if this is so, within predator contexts the difference between A-calls and B-calls becomes hard to analyze.

It is immediately clear that the lexical contribution of B-calls must be extremely weak, since they appear both in non-predation $((2) \mathrm{d})$ and in predation $((1) \mathrm{b}, \mathrm{c}, \mathrm{d} ;(2) \mathrm{d})$ contexts; furthermore, within predation contexts they occur both in eagle ((1)b) and in leopard ((1)c, d) contexts, and in contexts in which the threat is on the ground ((1)b) as well as in the canopy $(((1) c)$. In addition, Cäsar et al. $2012 \mathrm{~b}$ note that "B-calls were also often produced in non-predatory contexts and sometimes in the absence of external events, especially when monkeys were descending or foraging close to the ground, when an observer was blocking their intended path, during inter-group encounters and, for unhabituated groups, in response to humans". We take these observations to argue for the very weak lexical specification in (7).

(7) B-call

$\mathrm{B}$ is applicable if and only if there is a noteworthy event.

Difficulties arise when we notice that the A-call also occurs in heterogeneous environments. While in our data A-calls are not used in non-predatory situations, within predator-related situations they occur both in 'raptor on the ground' situations ((1)a) and in 'cat in the canopy' situations ((1)c). It is hard to see what these two situations could have in common besides being situations of predation. So it would seem reasonable to posit the lexical specification in (8):

\section{(8) A-call (1st try)}

A is applicable if and only if there is a predator-related alert.

It is immediate that the A-call entails the B-calls, since if there is a predator-related alert, there is a a noteworthy event.

Although A- and B-calls have different lexical specifications according to the rules in (7) and (8), within predator contexts it is entirely unclear what could distinguish them from each other. With the device of 'monkey scalar implicatures' of Schlenker et al. 2014, to appear a, b, we could try to add 
to our analysis a pragmatic component as in (9), with the prediction in (10). In essence, the idea is that A-calls are more informative than B-calls and thus are used whenever possible.

(9) Competition among calls

a. A- and B- calls compete with each other for informativity.

b. Since A is more informative than B, if a B-call is produced, infer that the A-call was not applicable at the same moment and in the same situation.

(10) Prediction of (9) given (7) and (8)

When pragmatic strengthening is applied, the B-call should only be applicable when there is a noteworthy event that is not a predator related alert (since in predator-related alerts the A-call can be used more informatively).

Given the lexical specifications in (7) and (8), this pragmatic component has an undesirable consequence: the B-call is predicted not to normally arise in situations of predation, as stated in (10). The heart of the matter is that while the A-call is more informative than the B-call, its lexical specification is still very weak. And since the competition rule in (9) has the effect of enriching the meaning of B with the negation of A, the result is an enriched meaning for B which is just too strong, as it is predicted to be inapplicable in situations of predation.

It was noted by Cäsar et al. 2013 that the first position in a sequence is somehow special, as it is often followed by a long pause, especially in non-stereotypical situations (raptor on the ground; cat in the canopy - see Cäsar et al. 2013, supplementary materials, figure 3S). Could we improve our analysis if we allowed calls to have different meanings in the first position and in the rest of sequences? As it turns out, this wouldn't help. For with respect to the first position, the B-call occurs both in situations of predation ((1)d) and in situations of non-predation $((2) d)$, which suggests that even relative to the first position it functions as a general call. And the problem we had with the Acall does not change a bit when we restrict attention to the first position, since we get an initial A-call both in 'raptor on the ground' and 'cat in the canopy' situations, which again suggests that it has a general predator-related function.

\section{Two Theories}

We believe there are two natural directions to explore to solve the problem we just laid out. One is to posit that the meaning of an entire sequence is not compositionally derived from the meaning of the individual calls it contains, but rather is obtained 'holistically' (Section 5.1). An alternative is to posit that each call has a constant individual meaning, but to rely more heavily on assumptions about the environmental context, by (i) making greater use of world knowledge in connecting literal meanings to their conditions of use, and by (ii) relativizing the meaning of a call to the precise moment at which it is uttered (Section 5.2). To be more specific: we will posit that (i) the A-call is a (serious) nonground alarm, and that the reason it occurs in 'raptor on the ground' situations is that even in these cases the threat (as opposed to the predator) is non-ground-related; and that (ii) the nature and the level of the threat can change as a sequence is produced - which will for instance connect the number of A-calls to the duration of the threat. Further theoretical possibilities are discussed in Section 5.3. 


\subsection{A non-compositional theory}

As mentioned, Arnold and Zuberbühler (2006, 2008, 2012, 2013) argued that Putty-nosed monkeys have pyow calls that serve as generals or attention getters, hack calls that are usually related to the presence of aerial threats, and pyow-hack sequences that are predictive of group movement. Arnold and Zuberbühler argued that pyow-hack sequences are syntactically combinatorial because they are made from individual calls; but that they are not compositional, because their meaning does not seem to be derivable from that of their individual parts. Importantly, pyow-hack sequences are made of a small number of pyows followed by a small number of hacks, but the number of calls of each sort appears to be flexible - and to have the same effect on group movement when the total number of calls is kept constant. Thus they showed in field experiments that the distance travelled was roughly the same when the female Putty-nosed monkeys heard a series of 3 pyows followed by 3 hacks, 1 pyow followed by 5 hacks, or 5 pyows followed by 1 hack. While one could posit highly disjunctive rules to cover all these cases, it seems more economical to posit that the semantics is not sensitive to the precise number of repetitions, and to give lexical specifications such as those in (11), where as before $X^{+}$stands for an arbitrary number of consecutive occurrences of call X. (Note that Arnold and Zuberbühler only argue that pyow-hack sequences should be given a non-compositional treatment, but once this device is available, one may extend it to pure pyow and pure hack series as well - which is why in (11)a, b we leave it open whether individual calls or entire call sequences should be given a meaning.)

(11) Putty-nosed calls: syntactic combination without semantic combination?

a. $\mathrm{P} / \mathrm{P}^{+}$series are used as general calls.

b. $\mathrm{H} / \mathrm{H}^{+}$series are usually associated with the presence of aerial threats.

c. $\mathrm{P}^{+} \mathrm{H}^{+}$sequences are usually predictive of group movement.

Given this precedent, we could try to extend the method of whole sequence meanings to Titi calls. As an initial attempt, we could take the context descriptions in (1) and (2) to come close to the meanings of entire sequences. On a technical level, we specify meanings for complete sequences (i.e. sequences that include all the calls uttered) for otherwise we would run into ambiguities, as it would not be clear whether a sequence $A^{++} B^{++}$should be analyzed as an $A^{++}$sequence followed by a $B^{++}$ sequence, or as a primitive $A^{++} B^{++}$sequence; by specifying meanings for complete sequences of a given form, the first option is precluded. ${ }^{12}$ To further refine the analysis, we may make use of rules of competition among complete call sequences in order to have a leaner analysis, for instance the one illustrated in (12)-(13).

(12) Non-compositional Titi meanings

If $s$ is a complete call sequence, then

a. if $\mathrm{s}$ of the form $\mathrm{B}^{++}, \mathrm{s}$ is applicable if and only if there is a noteworthy event.

\footnotetext{
${ }^{12}$ As was mentioned in connection with the generalizations in (1) and (2), our data - including those reproduced in the Appendix - are based on the first 30 calls of any sequence. But after the $30^{\text {th }}$ call, the patterns obtained with $\mathrm{A}$ and $\mathrm{B}$ calls just repeat the end of the 30-call sequence, hence our generalizations are unaffected by the restriction to the first 30 calls.
} 
b. if $\mathrm{s}$ of the form $\mathrm{A}^{++}$, $\mathrm{s}$ is applicable if and only if there is a non-ground predator.

c. if $s$ of of the form $\mathrm{A}^{++} \mathrm{B}^{++}, \mathrm{s}$ is applicable if and only if there is a non-ground predator on the ground.

d. if $\mathrm{s}$ of of the form $\mathrm{AB}^{++}$, $\mathrm{s}$ is applicable if and only if there is a ground predator in a non-ground position.

\section{(13) Complete sequence competition}

a. A Titi sequence competes with all sequences obtained by replacing, call for call, any number of A's with (the same number of) B's and any number of B's with (the same number of) A's.

b. If a sequences $S$ is uttered and competes with a more informative sequence $S^{\prime}$, one can infer that $S^{\prime}$

wasn't applicable.

The (modest) benefit of this analysis is that it makes it possible to assign very simple meanings to the $\mathrm{B}^{++}$and $\mathrm{A}^{++}$series, with the pattern of precedence illustrated in (14). The point is that the competition principle in (13) will lead to an enrichment of these simple meanings by way of inferences that $\mathrm{A}^{\mathrm{n}}$ and $\mathrm{A}^{\mathrm{k}} \mathrm{B}^{\mathrm{m}}$ could not have been used; the results are illustrated in (15).

(14) Informativity relations among Titi sentences that are alternatives to each other

according to (12) (higher $=$ strictly more informative) for any $\mathrm{n} \geq 3$ and for any $\mathrm{k}, \mathrm{m} \geq 2$ such that $\mathrm{k}+\mathrm{m}=\mathrm{n}$

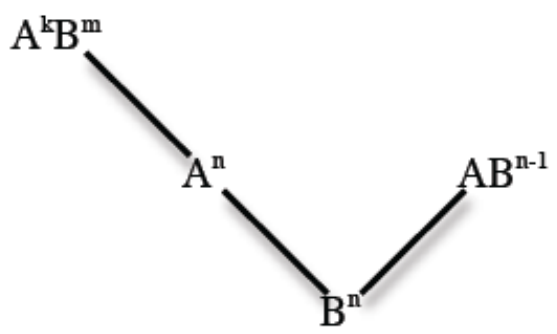

(15) Strengthened Titi meanings

For any $\mathrm{n} \geq 3$ and for any $\mathrm{k} \geq 1$ and $\mathrm{m} \geq 2,{ }^{13}$

a. for any sentence $S$ of the form $\mathrm{S}=\mathrm{B}^{\mathrm{n}}, \mathrm{S}$ can be used

if and only if there is an alert but no non-ground predator (or else $\mathrm{A}^{\mathrm{n}}$ would have been used) and no ground predator in a non-ground position (or else $\mathrm{AB}^{\mathrm{n}-1}$ would have been used),

if and only if there is a ground predator in a ground position;

b. for any sentence $S$ of the form $\mathrm{S}=\mathrm{A}^{\mathrm{n}}, \mathrm{S}$ can be used

if and only if there is a non-ground predator but not a non-ground predator on the ground (or else $\mathrm{A}^{\mathrm{k}} \mathrm{B}^{\mathrm{m}}$ would have been used),

if and only if there is a non-ground predator in the canopy;

c. for any sentence $S$ of the form $\mathrm{S}=\mathrm{A}^{\mathrm{k}} \mathrm{B}^{\mathrm{m}}, \mathrm{S}$ can be used iff there is a non-ground predator on the ground;

d. for any sentence $S$ of the form $\mathrm{S}=\mathrm{AB}^{\mathrm{n}}, \mathrm{S}$ can be used iff there is a ground predator in a non-ground position.

Still, we believe that this theory has three main flaws.

- Explanatory power: The analysis as it stands re-states in slightly improved form (due to mechanisms of competition) the generalizations we saw in (1). A symptom of this problem is that it has nothing to say about sequences whose form is slightly different from that of the sequences listed in (12). Thus this analysis lacks explanatory force.

${ }^{13}$ The conditions on $\mathrm{k}$ and $\mathrm{n}$ are more stringent in (14) than in (15) because in (14) we wish to guarantee that $\mathrm{A}^{\mathrm{k}} \mathrm{B}^{\mathrm{m}}$ has the right number of calls to serve as a competitor to $\mathrm{A}^{\mathrm{n}}$ and to $\mathrm{B}^{\mathrm{n}}$; since competitors are defined in (13) by call-for-call replacement, this leads to the condition the condition $\mathrm{k}+\mathrm{m}=\mathrm{n}$. 
- Initial reactions: Cäsar 2012a notes that after hearing an A-call, the Titi monkeys very quickly start looking upwards, as shown in (16) and (17) (latency is computed relative to the start of the playback). Presumably this fast reaction is also triggered in $\mathrm{A}^{++} \mathrm{B}^{++}$sequences, which according to (12)c provide information about non-ground predators that are on the ground. Of course it might be that $A^{++} B^{++}$ sequences are less common than sequences with an initial A that $d o$ pertain to non-ground threats; if so, looking up upon hearing an initial A-call might still be a good strategy. But as we will soon see, a leaner theory can be obtained if we assume that the initial reaction of looking up directly reflects the semantic content of A-calls.

(16) 'Looking upwards' as a reaction to A- vs. B-calls (from Cäsar et al. 2012a figure 3)

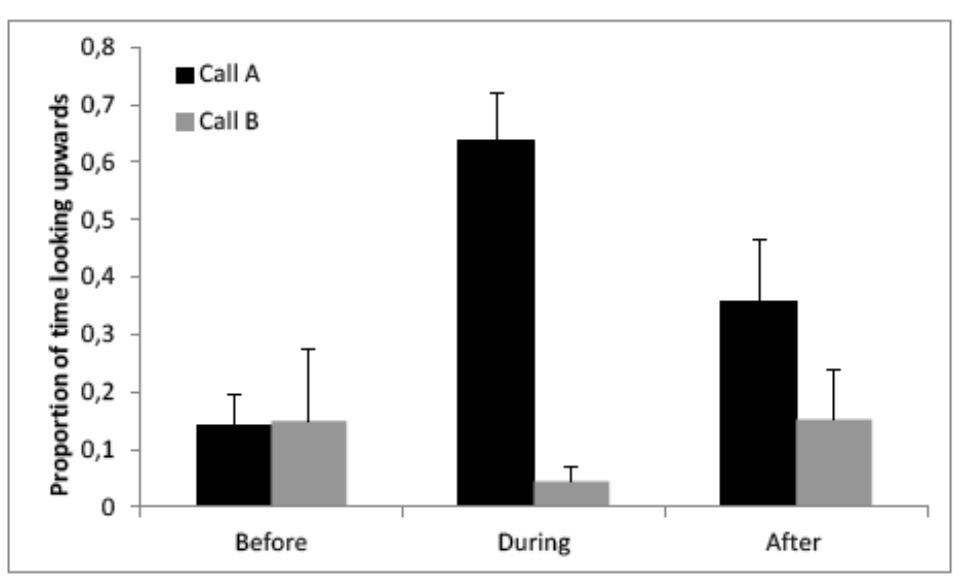

(17) Latency of A- vs. B-calls (from Cäsar et al. 2012a figure 2; latency is computed after the start of the playback)

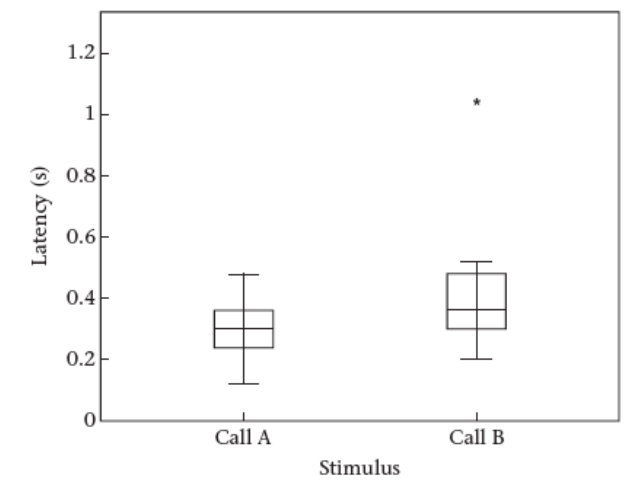

Figure 2. Box plots indicating the latencies in response to the different playback types. The box plots show the median and 25 th and 75 th percentiles; the whiskers indicate the values within 1.5 times the interquartile range, and the
extreme case. Number of trials: 10 for call A and 12 for call B.

- Timing: Titi sequences are not very fast, with an average inter-call interval of 1.4s. (12)b-c above posits distinct meanings for $\mathrm{A}^{++}$vs. $\mathrm{A}^{++} \mathrm{B}^{++}$sequences in order to explain why $\mathrm{A}^{++}$is found in 'raptor in the canopy' situations while $\mathrm{A}^{++} \mathrm{B}^{++}$is found in 'raptor on the ground' situations. It is thus clear that one must wait at least until one has heard a $\mathrm{B}$-call to tell that one is hearing an $\mathrm{A}^{++} \mathrm{B}^{++}$rather than an $\mathrm{A}^{++}$sequence. But in 'raptor on the ground' situation, when B-calls are produced, the first B-call appears on average after the 12th position in the sequence (average position: 12.6); very roughly, this gives an average waiting time of $16-17 \mathrm{~s}$ or so before one hears the first B-call after the first call is 
produced.$^{14}$ This would seem like a very long time to wait to determine which elementary meaning one is exposed to - hence a theoretical dilemma. If the receivers of the message cannot start reacting before they have determined which of the two sequence types they are hearing, how can such a meaning assignment be adaptive ${ }^{15}$ Alternatively, it could be that the informational contents of $\mathrm{A}^{++} \mathrm{B}^{++}$and $\mathrm{A}^{++}$sequences license the same initial reaction - so that the receiver need not decide which sentence it is hearing before starting to react. But if so, shouldn't we take that initial reaction to provide a key to the semantic content of the initial part of the sequence within a (more) compositional theory? This is what we will propose in our compositional theory, with the claim that A-calls call attention to a serious non-ground threat.

We conclude that the non-compositional analysis of Titi sequences has significant flaws, and that an alternative theory should be explored.

\subsection{An alternative}

We will now circumvent the problems we encountered with our initial specifications of lexical meanings by assuming (i) that the B-call is highly underspecified, as assumed earlier; (ii) that the Acall does not provide information about the nature of the predator and its location, but rather about the appropriate reactions to the relevant threat - with some non-trivial assumptions about the connection between the threats and the appropriate reactions to them; (iii) that the calls are produced as individual units, with the result that a call reflects the state of the environment at the precise time at which it is uttered; and (iv) that the total informational content of the sequence is the conjunction of the claims made by the individual calls at the relevant times' (we revisit this possibility in Section 5.3). As argued before, we take (i) to be particularly natural in view of the very broad distribution of the B-call. We will use the behavioral data discussed at the end of Section 5.1 (= A-calls trigger a reaction of 'looking up') to motivate an analysis of the A-call as a 'non-ground alert' call; theories that do not have this 'non-ground' component would have to explain these reactions in a more roundabout way. In addition, we take (iii)-(iv) to be the simplest possible assumptions about call composition: in effect, each call is taken to contribute its informational content independently from the others, which is natural in view of the slow time course of Titi sequences. We take these four assumptions to be rather close to a 'null' theory of Titi call interpretation.

\footnotetext{
${ }^{14}$ This is a rough approximation, obtained by multiplying $\mathrm{N}$ by $\mathrm{L}$, with $\mathrm{N}=$ the average number of inter-call intervals heard between the first call of the sequence and the first B-call hence $\mathrm{N}=12.6-1=11.6$, since the first $\mathrm{B}$-call appears on average in position 12.6;

$\mathrm{L}=$ inter-call average length computed over all sequences $(=1.437 \mathrm{~s})$.

The result is $\mathrm{N} \times \mathrm{L}=16.7 \mathrm{~s}$.

${ }^{15}$ Note that the lexical meaning of $\mathrm{A}^{++}$is weaker than (and hence compatible with) that of $\mathrm{A}^{++} \mathrm{B}^{++}$. One could thus argue that the informational component these two sequence types have in common licenses the appropriate reaction as long as the ambiguity has not been resolved. But our analysis crucially hinges on the fact that the lexical meaning of $\mathrm{A}^{++}$is pragmatically enriched with the negation of $\mathrm{A}^{++} \mathrm{B}^{++}$. For behavioral purposes, it is this enriched meaning that presumably matter. But the enriched meaning of $\mathrm{A}^{++}$meaning is clearly incompatible with that $\mathrm{A}^{++} \mathrm{B}^{++}$, which means that the proposed strategy won't help much.
} 
To be more specific, we will assume (using (ii)) that the A-call is specified for serious nonground alerts, and that the reason we find an initial $\mathrm{A}^{++}$sequence in 'raptor on the ground' situation is that raptors attack by flying, and thus that even when a raptor is on the ground the threat isn't a ground one. As for the fact that $\mathrm{B}^{++}$sequences can follow $\mathrm{A}^{++}$sequences, this will be taken (using (iii)-(iv))) to reflect the fact that in some cases the threat level is taken to diminish after the appearance of an initial trigger, and thus that the 'serious non-ground alert' content of the A-call stops being applicable after a while, leaving B as the only contender. Thus the fact that the A-call is specified for a serious non-ground alert will matter in that part of the analysis - and in this respect we make an assumption that departs from a completely 'null' theory (since the 'non-ground' component is motivated by behavioral data, but the 'serious' component is justified by more theory-internal considerations).

To develop the analysis in greater detail, we will need three assumptions that have some independent motivation (but which should of course be further assessed in future research).

(18) a. A raptor hunts by being perched or by flying; a raptor on the ground is not in a hunting position.

b. Cats become less dangerous once they have been detected (because they are ambush rather than pursuit hunters).

c. Capuchins are dangerous even if they have been detected (because they are pursuit hunters).

About (18)a: one can infer from descriptions of eagle hunting techniques to hunt monkeys that they attack from above, not from the ground (see Shultz and Thomsett 2007 for eagles in the Tai forest ${ }^{16}$ ). About (18)b: an example from a different part of the world is given in Zuberbühler et al. 1999, who show "after detection and high alarm call rates" a radio-collared leopard "gave up its hiding location and left the group significantly faster than would be expected by chance", which would suggest that detection was sufficient to considerably lower its chances of success (see also Zuberbühler and Jenny 2007).

About (18)c: Fedigan 1990 describes hunting strategies by Capuchin monkeys which rely on chasing a prey.

Our semantics will be extremely simple: each call will individually contribute a simple meaning to a sequence. Importantly, however, we take into account the fact that call rates are relatively slow, and thus that different calls provide information about the environment at different times. For this reason, the applicability conditions of calls are relativized to utterance times. It should be noted that a side effect of this relativization to times is that repetition of calls is not semantically vacuous: each repetition of an alarm call makes a new claim, namely that the relevant alarm holds at the time of utterance of that call.

On this view, successive calls are separate sentences making claims about different moments, as stated in (19). (19)a is just the statement that B is a general call. (19)b encodes the treatment of A as a serious non-ground alert.

\footnotetext{
${ }^{16}$ The authors describe both a 'searching' and a 'sit-and-wait' strategy, but it seems clear that even the latter involves attacks from above (as the authors write, the predators 'drop down' on the monkeys).
} 
(19) Semantics of Titi calls (partial: A- and B-calls only)

For any time $t$,

a. B uttered at $t$ is true if and only if there is a noteworthy event at $t$.

$b$. A uttered at $t$ is true if and only if there is a serious non-ground alert at $t$.

(20) Semantics for sequences

A call sequence is true just in case each call it contains is true at the time of that call's utterance.

Two examples are given in (21).

(21) Two examples

a. B uttered at time 0 is true if and only if there is a noteworthy event at time 0 .

b. $A B$ uttered at time 0 for $A$ and at time 1 for $B$ is true if and only if

A uttered at time 0 is true and $B$ uttered at time 1 is true, i.e. if and only if

there is a serious non-ground alert at time 0 and there is a noteworthy event at time 1 .

(21)a is unexceptional; (21)b displays a simple case in which the two calls are evaluated at different times: time 0 for $\mathrm{A}$, time 1 for $\mathrm{B}$.

It is immediately clear that the distribution of B-calls must be further constrained - for without addition, the semantics would lead one to expect that the B-call can arise in all situations, contrary to fact. The natural solution is to adopt the rule of competition we discussed in (9).

(22) Pragmatics of Titi calls

a. A- and B-calls compete with each other following the rules in (9).

b. Derived result: if B is uttered at time t, one can infer that at time $t$ (i) there is a noteworthy event, but not

(ii) a serious non-ground alert (or else the A-call would have been used).

Importantly, we do not get the undesirable result we had in our initial discussion, where A was specified as a predator call and B was thus incorrectly strengthened into a non-predator call. The strengthening we now obtain is far more adequate: $\mathrm{B}$ should not be uttered at time $\mathrm{t}$ if there is a serious non-ground alert at that time.

Let us now see how our hypotheses can derive the patterns we observe. Let us start with the data in (1)-(2), repeated in condensed form in (23).

(23) Simplified Generalizations

\begin{tabular}{|c|c|c|c|c|c|}
\hline & & a. Raptor & b. Cat & c. Capuchin & $\begin{array}{l}\text { d. Non-predator } \\
\text { related }\end{array}$ \\
\hline \multirow[t]{2}{*}{ Experimental } & Canopy & $\begin{array}{l}\text { (i) } \mathrm{A}^{++}(\text {mean }=26.8 \\
\text { calls) }\end{array}$ & (i) $\mathrm{AB}^{++}$ & & \\
\hline & Ground & $\begin{array}{l}\text { (ii) } \frac{\mathrm{A}^{++} \mathrm{B}^{++}}{\text {number of } \mathrm{A}^{\prime} \text { 's: } 2.2 \text { ) }} \\
\text { (a) }\end{array}$ & (ii) $\mathrm{B}^{++}$ & & \\
\hline Naturalistic & & $\begin{array}{l}\text { (iii) Flying: } \\
\mathrm{A}^{+}(\text {mean }=2.2 \text { calls) } \\
\text { (iv) Calling or } \\
\text { perched: } \\
\mathrm{A}^{++} \quad \text { (mean }=15.8\end{array}$ & & $\begin{array}{l}\text { In tree: } \\
\mathrm{A}^{++}, \mathrm{A}^{++} \mathrm{B}^{++} \mathrm{A}^{++} \text {, etc, } \\
\text { with } \quad \mathrm{C} \text {-calls } \\
\text { interspersed } \quad \text { (see } \\
\text { Appendix) }\end{array}$ & $\begin{array}{l}\text { deer, } \\
\text { foraging/descending } \\
\mathrm{B}^{++}\end{array}$ \\
\hline
\end{tabular}

Raptor situations: The generalizations (23)a(i), (iii) and (iv) are unsurprising in view of our hypotheses about eagle hunting techniques in (18)a. First, a model raptor in the canopy or a perched raptor present serious non-ground threats, and the threats should be taken to persist in time since these are typical hunting positions - hence the fact that the series are long. Second, series of A-calls are 
shorter in the naturalistic 'flying raptor' situations in (23)a(ii) (Mean number of A's: 2.2) than in the naturalistic 'calling/perched raptor' (M=15.8 A's, $W=13, p=.00021)^{17}$ situations in (23)a(iv) or in the experimental 'raptor in canopy' situations in (23)a(i) $(\mathrm{M}=26.8, W=0, p=.00048)$; this is presumably because a raptor that flies away is a briefer threat than the immobile raptors in typical hunting position (it might also be that in experimental situations the model raptor remains perched longer than in naturalistic ones, although it might become clear at some point that it is not a normal living raptor). ${ }^{18}$ Finally, the pattern in (23)b(ii) is expected if we remember that a raptor on the ground will attack (if it does) by flying, as stated in (18)a. Therefore the initial $\mathrm{A}^{++}$we find is unsurprising and provides the most urgent message first, as a danger may be coming from above.

Since immobility on the ground is not a typical hunting position, it is also unsurprising that after a while the threat stops being considered as serious - presumably because a raptor would not normally remain motionless on the ground for long periods of time. As mentioned above, the first Bcall in 'raptor on the ground' situations occurs on average in position 12.6 in the sequence - with a possible estimate of $16-17 \mathrm{~s}$ after the first call. This might give enough time to the caller to decide that the threat isn't too serious any more; if so, it is because the A-call is specified for serious non-ground threats that it stops being applicable after that time, with the result that B-calls start being used instead. By the logic of pragmatic competition, this is the only case in which we see B-calls for raptor-related threats.

Cat situations: In 'cat on the ground' situations, only the B-call can be used, hence the B-series in (23)b(ii). The production of an A-call (specified for a 'serious non-ground alert') at the beginning of 'cat in the canopy' situations should give us pause. Given our assumptions, it can be explained:

-When a cat is detected in the canopy, it represents a serious non-ground threat, hence the production of an A-call.

- As a consequence of this A-call, it can be assumed that the cat has been detected by conspecifics. As a result, the threat level diminishes, in accordance with (18)b. Because the A-call is specified for serious non-ground threats, it can't be used any more, with the result that only the B-call can be used (we come back in the conclusion to the cognitive implications of this reasoning).

Capuchin situations: Naturalistic capuchin situations give rise to a diversity of calling sequences, some of them with quite a few A-calls. This is strikingly different from the stereotyped $\mathrm{AB}^{++}$ sequences we found in 'cat in the canopy' model experiments. It is thus notable that two mammal

\footnotetext{
${ }^{17}$ We used Mann-Whitney tests using each call as an independent data point. The structure of the data forces us to merge all groups into one for these analyses, rather than studying a possible group effect (there is no group for which there is more than 3 calls for two of the situations). But we have no reason to believe that there exists such a group effect.

${ }^{18}$ There are fewer calls in (naturalistic) 'calling/perched raptor' than in (experimental) 'raptor in the canopy' situations $(W=11.5, p=.16)$. If this difference were significant, it could be because in the former case the trigger disappears more quickly than in the second, which involves raptor models.
} 
predators in non-ground situations give rise to such different calling behaviors. Now one source of the difference might be that real capuchins move in ways that model leopards don't. ${ }^{19}$ But an additional explanation might lie in the difference between leopard and capuchin hunting strategies outlined in (18)b,c: capuchins continue to be dangerous even after they have been detected, hence we have no reason to expect the $\mathrm{AB}^{++}$pattern we found in 'cat in the canopy' situations. While this doesn't explain the details of the complex patterns we find in capuchin situations, it does give us a way to address an initially surprising difference.

Non-predatory situations: It is clear that in situations that do not involve serious threats the A-call cannot be used, hence the fact that we only find B-calls in these cases.

(We leave for future research an analysis of the $\mathrm{C}$-call, which might conceivably be a 'group movement' call - but it is too early to make precise pronouncements.)

\subsection{Further possibilities}

Throughout Section 5.2, we assumed that the global content of sequences is obtained by taking the conjunction of the claims made by the individual calls about the times at which they are uttered. One could depart from this assumption and allow for a mechanism of 'self-correction', as suggested by an anonymous referee. In view of our analysis of the B-call as highly underspecified, this won't change things when only the literal meaning of the calls is considered, since the A-call entails the B-call with the result that the two couldn't contradict each other to begin with. The situation changes when the strengthened meaning of the B-call is taken into account, since by (22)b it will then be tantamount to $B$ and not $A$. But we already posited that calls are evaluated with respect to the precise time at which they are uttered, so that no contradiction need arise between the utterance of an A-call at the beginning of a sequence and the later appearance of a B-call, interpreted on its strengthened meaning, in the same sequence. Concretely: we took the $\underline{\mathrm{A}^{++} \mathrm{B}^{++}}$sequences found in 'raptor on the ground' situations $(=(23) \mathrm{a}(\mathrm{ii}))$ to be due to the fact that a raptor of on the ground triggers a serious nonground alert (because it would attack by flying), which then becomes less serious (because the raptor is not in a typical hunting position) - and this, in turn, licenses the strengthened meaning of the $\mathrm{B}$-call ('B and not $\mathrm{A}^{\prime}$, so to speak). Similarly, the $\mathrm{AB}^{++}$sequence found in 'cat in the canopy situations' (= (23)b(i)) were explained by the initial presence of a serious non-ground alert, downgraded to a less serious alert once the cat could be taken to have been detected - which licensed the strengthened (i.e. 'B and not $\mathrm{A}^{\prime}$ ) meaning of the B-call. We conclude that (i) an analysis in terms of self-correction

\footnotetext{
${ }^{19}$ Note however that some naturalistic situations give rise to stereotyped sequences, as can be seen in the naturalistic Eagle-related sequences in (23)a(iii)-(iv).
} 
would not bring any result that we don't already derive, and that (ii) it would thus needlessly complicate the theory. ${ }^{20}$

\section{Conclusion}

Our findings are decidedly deflationary: we did not uncover any non-trivial syntactic or semantic properties in Titi call sequences. As a result, our findings do not yield new insights into the evolution of human language, although a detailed typology of primate languages will certainly prove essential to this long-term endeavor. Besides contributing to this typology, our study shows that methods from contemporary linguistics can play an important role in mapping the space of possible theories. In addition, we obtained substantive (if tentative) findings pertaining to division of labor between semantics, pragmatics and the environmental context, as well as some questions for future research. ${ }^{21}$

\subsection{Semantics/pragmatics vs. the environmental context}

The present study contributes to the general program of applying explicit formal methods to the semantics of call sequences, but it also highlights the importance of assumptions about the environmental context. With our initial hypothesis that calls directly convey information about predator type and/or predator location, we were unable to provide a coherent meaning for A- and Bcalls, and we had to resort to a somewhat unappealing theory in which entire call sequences have a non-compositional meaning. Importantly, this negative conclusion could only be reached because we were very explicit about the underlying semantics and pragmatics, and thus methods from contemporary linguistics were essential to that part of the analysis. On the positive side, we believe that a better and simpler theory can be obtained if we assume that each call is produced and interpreted independently, and if we make use of more sophisticated assumptions about the

\footnotetext{
${ }^{20}$ An anonymous referee suggests that the A-call could be analyzed as contributing the information that there is 'danger in the air', whereas the B-call could be specified for the presence of a 'remarkable animal on the ground'. For the B-call, the referee's proposed specification is clearly stronger than our highly underspecified lexical entry; it would fail to account for the fact, mentioned above, that B-calls occur in situations in which no animal has been detected (Cäsar et al. 2013; see also the sample data in the Appendix). For the A-call, the precise relation between the referee's analysis and ours depends on how literally 'danger in the air' is interpreted. One could take 'danger in the air' to mean that there is a dangerous entity in the air - and if so a mechanism of correction would be needed for $\mathrm{A}^{++} \mathrm{B}^{++}$sequences produced in 'raptor on the ground' situations, as these do not involve a dangerous entity in the air. Alternatively, we could take 'danger in the air' to correspond to what we called 'non-ground alert' (without the 'serious' component added in (19)b) - with the possibility that this is triggered in 'raptor on the ground' situation because the danger (though not the predator) is non-ground. Either way, this analysis must explain why the observed sequences transition from A to B in 'cat in the canopy' situations $\left(\mathrm{AB}^{++}\right)$and in 'raptor on the ground' situations $\left(\mathrm{A}^{++} \mathrm{B}^{++}\right)$. The referee suggests that these are corrections, and that the transition is immediate in 'cat in the canopy situations' so as to 'quickly make clear that the danger is considerably weakened'. This analysis has several drawbacks, however. (i) As mentioned, it posits an incorrect meaning for the B-call. (ii) It must explain which cases of call concatenation are conjunctive, and which are corrective. (iii) It takes Titis to produce false information in 'cat in the canopy' situations, since these contain a $\mathrm{B}^{++}$sub-sequence, in the absence of any 'remarkable animal on the ground' (and no self-correction follows in this case).

${ }^{21}$ See Schlenker et al. to appear b for a survey of several recent studies in primate linguistics, with a more systematic discussion of the division of labor among different modules (notably semantics, pragmatics, and the environmental context).
} 
environmental context. Two proved particularly crucial: first, we assumed that a raptor on the ground still signals the presence of a non-ground threat; second, we assumed that the meaning of calls is relativized to the precise time at which they are uttered, with the result that the composition of a sequence sometimes reflects the way in which the environmental context changes as the sequence is uttered. This result can in part be seen as a cautionary tale about the analysis of monkey sequences: not everything that displays complex syntactic or semantic patterns should be analyzed with complex syntactic or semantic rules; in this case, the complexity of the data can be derived from the interaction of simple semantic rules and plausible assumptions about the environmental context.

\subsection{The role of competition among calls}

It should be added that besides semantics and the environmental context, pragmatic competition among calls played an important role in our explorations, since this mechanism was crucial to explain why B-calls are not found in all situations. Thus the general issue of the division of labor between semantics, pragmatics and environment/world knowledge turned out to be the main issue in this study - as it was (in a different way) in Schlenker et al.'s (2014) study of male Campbell's monkey calls (see also Schlenker et al. to appear a for related discussions about Putty-nosed monkey pyow-hack sequences, and Schlenker et al. to appear $b$ for further applications of the same methods). It would be particularly interesting in future studies to (i) assess more direct evidence in favor or against rules of pragmatic competition, and possibly (ii) explore the cognitive/neural underpinnings of such rules. ${ }^{22}$

\subsection{Meaning and cognition}

Finally, we should ask what the present analysis can tell us about Titi monkey cognition.

- While in human language implicatures were taken in the neo-Gricean tradition to arise from principles of communicative rationality (Grice 1975, Horn 1972), far less is needed to obtain the desired mechanism of meaning enrichment. All we need is a rule whereby a monkey should produce the most specific call compatible with its epistemic state, without necessarily reasoning on the hearer's epistemic state - a point already made in Schlenker et al. 2014 (see fn. 10). ${ }^{23}$

\footnotetext{
${ }^{22}$ Further afield, one could seek to assess the existence of such rules in animals acquiring lexical meanings that can be experimentally modulated (e.g. dogs or parrots; see for instance Kaminski et al. 2004, Pepperberg 2010). Specifically, one could expose such animals to two labels $L$ and $L^{\prime}$ and a learning environment in which $L^{\prime}$ is true in a strict subset of the situations in which $L$ is true (so that $L^{\prime}$ is strictly stronger than $L$ ). One could then test whether $L$ blocks $L^{\prime}$ when both are applicable, be it in comprehension or (if testable) in production.

${ }^{23}$ Two remarks should be added.

(i) It was once objected to us that Titi monkeys couldn't have the cognitive abilities to derive implicatures, which human 4-year-olds have trouble understanding in human language. While we think it dubious to draw inferences on distant animals on the basis of human data, it is certainly legitimate to ask about the cognitive underpinnings of the mechanisms we posit. Several remarks are in order in this connection. First, as already mentioned, our rule of competition among calls need not rely on a theory of mind. Second, the comparison with human children is misleading along several dimensions: the child data we know of display problems with implicatures in perception, but they do not (yet) show that children produce under-informative sentences which would be the right point of comparison for the production data discussed here. More importantly, recent results show that human children do derive implicatures under several conditions, for instance with numerals
} 
- Our analysis of sequences triggered in 'cat in the canopy' situations could be taken to have stronger implications. We posited that the presence of a cat in the canopy licenses an initial A-call, indicating the presence of a 'serious non-ground alert'. We then reasoned that after this initial A-call, the cat can be taken to have been detected, hence a lowering of the threat level and the appearance of B-calls. But this reasoning is non-trivial, as it must take into effect the epistemic effects of the utterance of an Acall. Whether this is indeed the cognitive mechanism at work here remains to be seen. ${ }^{24}$

(Papafragou and Musolino 2003), or when alternatives are made salient (Barner et al. 2011). Finally, one might be rather surprised by the cognitive abilities of some New World monkeys. For instance, capuchin monkeys have displayed a sophisticated behavior in tasks pertaining to metacognition, decision-making, and deception, among others (see Parrish and Brosnan 2012 for a review). No comparable data exist for Titis, but caution is in order.

(ii) The 'grammatical' (or 'localist') approach developed by Chierchia et al. 2012 also takes implicatures to be derived without a theory of mind, but for a different reason. Chierchia et al. argue that implicatures can be derived at the level of constituents rather than just at the level of complete utterances, and they analyze them by way of covert exhaustivity operators akin to only, which are unpronounced but nonetheless present in the syntax. Since we take individual calls to form complete utterances, this type of argument is not applicable here. Furthermore, the enrichment mechanism we posit could in principle be adopted within standard ('globalist') neo-Gricean theories of implicatures - at the cost of sacrificing the fine-grained interaction between the derivation of implicatures and the precise state of mind of the speaker and addressee.

${ }^{24}$ Two points should be added.

1. As things stand, our analysis relies on the principle stated in (i):

(i) at time t, monkey $\mathrm{M}$ warns monkeys $\mathrm{M}^{\prime}$ with an $\mathrm{A}$ call in reaction to a cat in the canopy $=>$ at time $\mathrm{t}+1, \mathrm{M}$ believes that $\mathrm{M}^{\prime}$ believe that there is a threat in the canopy (and hence $\mathrm{M}$ believes that the cat has been detected)

(i) requires an ability to compute the effects of a vocal signal on another monkey's epistemic state. Crockford et al. 2012 argued that chimpanzees may take into account the epistemic effect of earlier calls (as well as of other cues) when producing alarms, thus uttering more calls towards ignorant audience members. Hattori et al. 2010 argued that under the right experimental conditions Capuchin monkeys take into account the epistemic state of their (human) audience (inferred through eyegaze cues, for instance) when performing certain requesting actions. We do not know of similar data pertaining to Titi monkeys, but preliminary observations suggest that Titis may change their sequences according to the presence of a conspecific (e.g. the approach of group member that was far away at the beginning of a sequence; Cäsar, personal observations; Cäsar, 2011).

Within our analysis, (i) above is essential to derive the result in (ii) below, which in turn explains why a cat in the canopy first licenses a serious non-ground alert at $\mathrm{t}$ (hence the A call), but soon thereafter only a non-serious alert (hence the $\mathrm{B}$ calls in $\mathrm{AB}^{++}$series). It is an open question whether we could derive (ii) on principled grounds if (i) turns out to be too strong.

(ii) at time t, monkey $\mathrm{M}$ warns monkeys $\mathrm{M}^{\prime}$ with an $\mathrm{A}$ call in reaction to a cat in the canopy $=>$ at time $\mathrm{t}+1$, the threat associated with the cat in the canopy is less serious than it was at $t$

2. Melissa Berthet (p.c.) reports that in ongoing field experiments, she replicates several of Cäsar's results, with one notable exception: in two sequences triggered in 'cat in the canopy' contexts, she obtains $\mathrm{B}^{++}$rather than $\mathrm{AB}^{++}$patterns. She hypothesizes that her cat models might have been less well hidden than in Cäsar's experiments, and that in the latter the Titis might initially have thought they were observing a raptor. From the present perspective, it might be that a conspicuous cat in the canopy is considered as a less serious threat than an inconspicuous one, hence a lower level of danger, and an initial B- rather than an initial A-call. But certainly our hypotheses should be revisited when Berthet's full data become available. 


\section{Appendix. Sample Sequences}

We provide below sample sequences obtained in various situations (up to the 30th call in each case). For the data obtained in field experiments with raptor and cat models, as well as for naturalistic raptor situations, and for situations of feeding/foraging/descending, we provide the first half of each block ${ }^{25}$. For naturalistic Capuchin situations and for non-predatory situations, we provide all our transcribed data. (We do not provide data from field experiments with tayra, puma and boa models, as these are the object of a separate study.)

Color code: $\quad$ A calls $=$ orange $; \mathrm{B}$ calls $=$ green $; \mathrm{C}$ calls $=$ yellow

口 Location experiments with Raptor vs. Cat Models (first half of each block)

-Raptor in the canopy

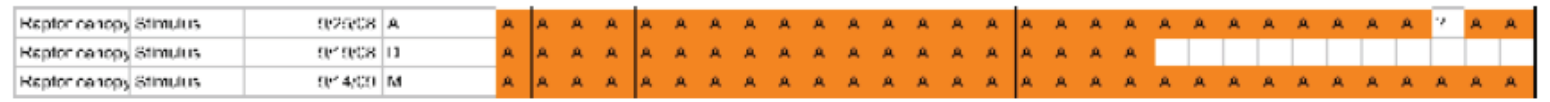

-Raptor on the ground

\begin{tabular}{|c|c|c|c|c|c|c|c|c|c|c|c|c|c|c|c|c|c|c|c|c|c|c|c|c|c|c|c|c|c|c|c|c|}
\hline Kepter gnarm stmulus & Fist: & $\mu$ & $\mu$ & a & a & $\mu$ & a & $\mu$ & a & a & $\mu$ & $\mu$ & a & $\mu$ & $\mu$ & 2 & $\mu$ & $\mu$ & a & $\mu$ & $\mu$ & a & $\mu$ & $\mu$ & B & H & H & \&: & c: & & в & $\mathrm{H}$ \\
\hline Repter gnurst stimulis & fin $24 \div 0$ & n & Aे & & & & & & & & & & & & & & & & & , & & & , & & & . & . & & & & & \\
\hline Kropter gaum strmulas & $\mathrm{H}^{2} / y^{\prime \prime}$ & ID & $\mu$ & h & h & $\mu$ & a & $\mu$ & $\mu$ & a & $\mu$ & $\mu$ & a & $\mu$ & $\mu$ & a & $\mu$ & $\mu$ & a & is & h & h & h & h & h & h & h & h & n & h & h & h \\
\hline Repoter gmarn stimulas & रำ & IS" & a & a & a & a & a & a & a & a & a & a & a & a & a & a & a & a & a & a & a & a & a & a & a & a & a & a & a & a & a & a \\
\hline
\end{tabular}

-Cat on the ground

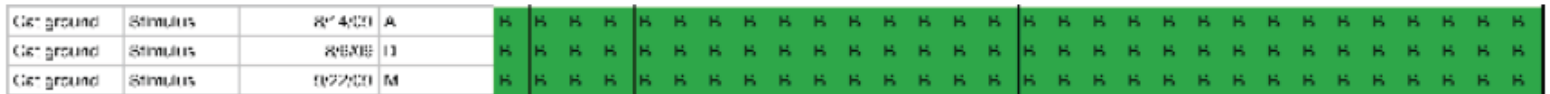

-Cat in the canopy

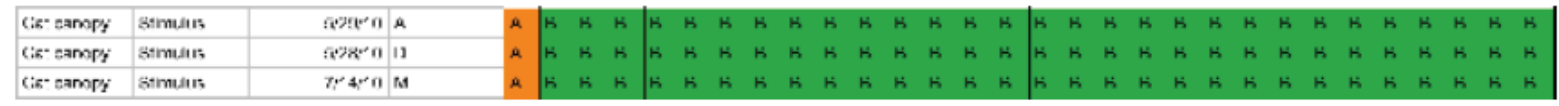

- Raw Data: Naturalistic Raptor Situations (first half of each block)

-Flying raptor

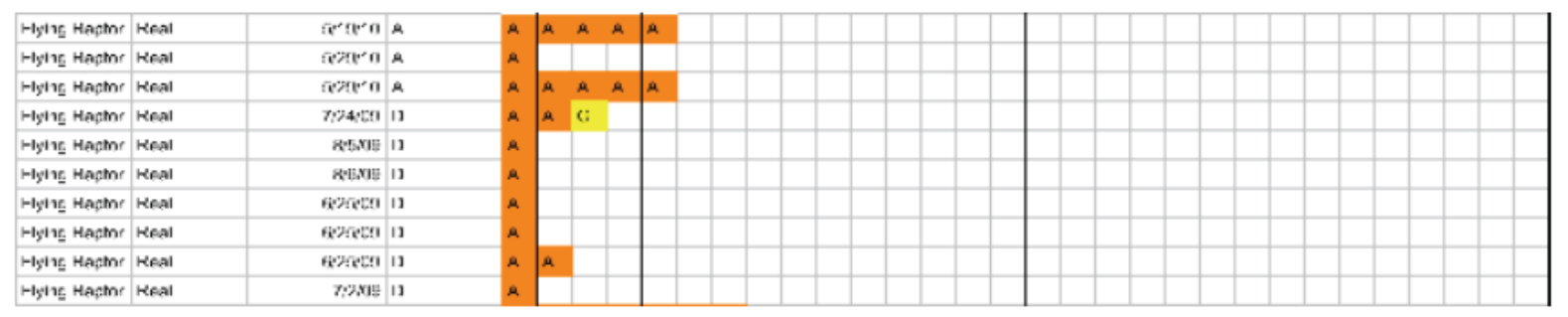

-Perched raptor

${ }^{25}$ Within blocks, sequences were ordered by alphabetical order (depending on the Titi group's name). 


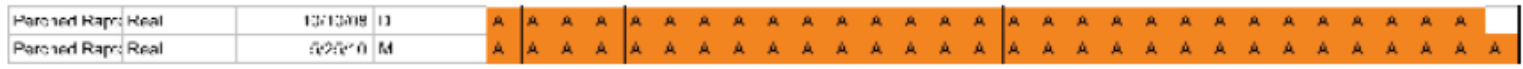

-Calling raptor

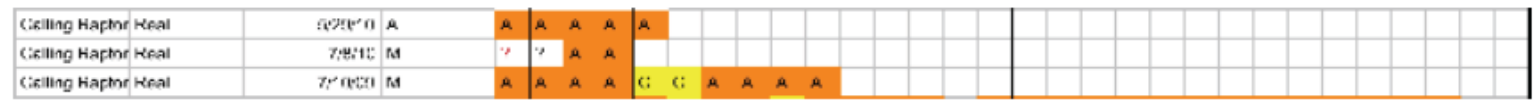

- Raw data: other animals (complete for Capuchins; samples for other animals)

-Puma

\begin{tabular}{|c|c|c|c|c|c|c|c|c|c|c|c|c|c|c|c|c|c|c|c|c|c|c|c|c|c|c|c|c|}
\hline PIma & Stmulas: & 11,5aly & a & \begin{tabular}{l|l} 
h & h
\end{tabular} & $\mathrm{B} B$ & h & $\mathrm{AS} \mathrm{R}$ & $\mathrm{B} \quad \mathrm{B}$ & $\mathrm{B}$ & $\mathrm{BH}$ & B B & $3 \mathrm{~B}$ & B & $\mathrm{B} \quad \mathrm{H}$ & $\mathrm{s}$ |r & $\mathrm{B} \quad \mathrm{B}$ & $3 \mathrm{~B}$ & $\mathrm{~B}$ & B & B & B $\quad \mathrm{r}$ & $\mathrm{B} \quad \mathrm{H}$ & 5 & H & H & h & h & B \\
\hline PIma & stmulus; & F: & 13 & h h h & $\mathrm{H} \quad \mathrm{H}$ & $\mathrm{H}$ & H H & S $\mathrm{H}$ & B & $\mathrm{H} H$ & H H & $5 \mathrm{H}$ & $\mathrm{H}$ & $\mathrm{H} \quad \mathrm{H}$ & H & $\mathrm{H} \quad \mathrm{H}$ & $\mathrm{H}$ & h & H & h + & H & $\mathrm{H} \quad \mathrm{H}$ & $B$ & $6 \mathrm{~h}$ & h & h & H & H \\
\hline PIma & stmusus & 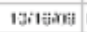 & M & \begin{tabular}{l|l} 
in & his
\end{tabular} & $\dot{B} \quad \mathrm{H}$ & h & $\mathrm{NO} / \mathrm{M}$ & mssilat & da $m c$ & $\mathrm{~cm}_{\mathrm{s}}-\mathrm{S}$ & - cac:al & 1) 25 & & & & & & & & & & & & & & & & \\
\hline
\end{tabular}

-Capuchin in tree

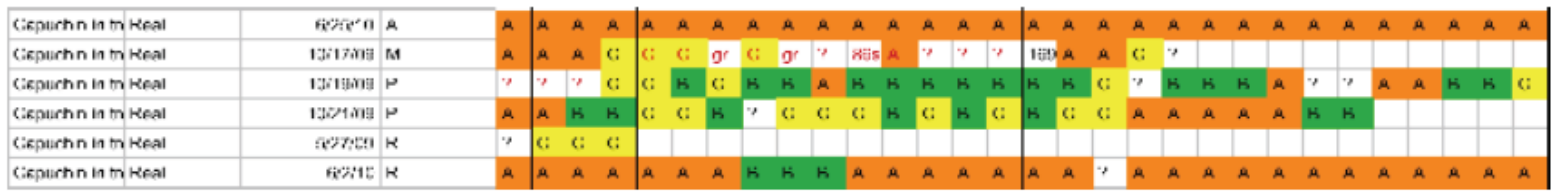

-Spotted cat on the ground

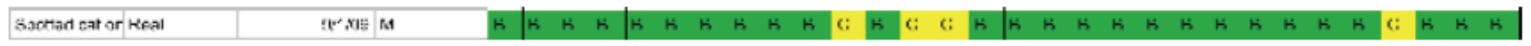

-Deer on the ground

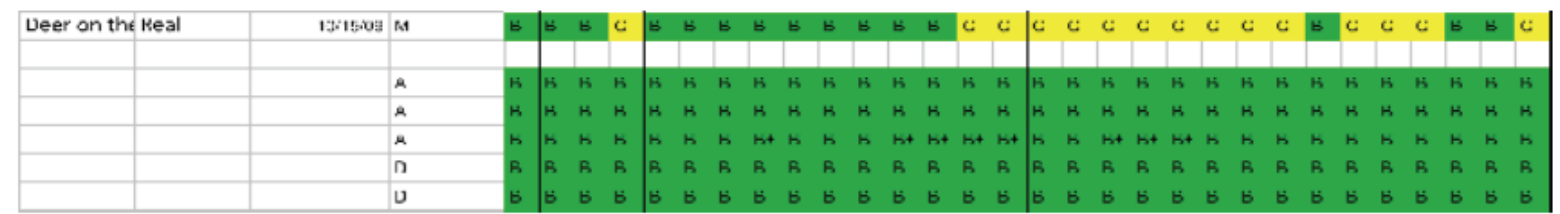

- Raw Data: feeding, descending, foraging situations (first half)

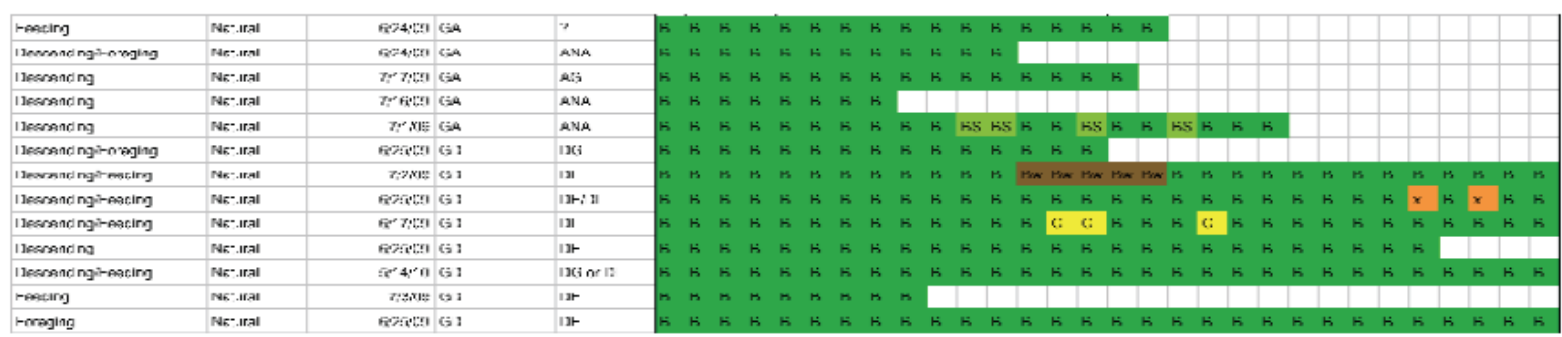




\section{References}

Arnold Kate, Pohlner Yvonne and Zuberbühler Klaus, 2001. Not Words but Meanings? Alarm Calling Behaviour in a Forest Guenon. Developments in Primatology: Progress and Prospects 35: 437-468.

Arnold Kate, Pohlner Yvonne and Zuberbühler Klaus, 2008. A forest monkeys alarm calls to predator models. Behavioral. Ecology and Sociobiology 62: 549-559.

Arnold Kate and Zuberbühler Klaus, 2006a. The alarm calling system of adult male putty-nosed monkey Cercopithecus nictitans martini. Anim. Behav. 72: 643-653.

Arnold Kate and Zuberbühler Klaus, 2006b. Semantic combinations in primate calls. Nature 441: 303.

Arnold Kate and Zuberbühler Klaus, 2008. Meaningful call combinations in a non-human primate. Curr. Biol. 18(5): R202-R203.

Arnold Kate and Zuberbühler Klaus, 2012. Call combinations in monkeys: Compositional or idiomatic expressions? Brain and Language 120(3): 303-309.

Arnold Kate and Zuberbühler Klaus, 2013. Female Putty-Nosed Monkeys Use Experimentally Altered Contextual Information to Disambiguate the Cause of Male Alarm Calls. PLoS ONE 8(6): e65660. doi:10.1371/journal.pone.0065660.

Barner, David, Neon Brooks \& Alan Bale, 2011. Accessing the unsaid: the role of scalar alternatives in children's pragmatic inferences. Cognition 188.87-96.

Cäsar Cristiane, 2011. Anti-predator behaviour of black-fronted titi monkeys (Callicebus nigrifrons). $\mathrm{Ph} . \mathrm{D}$ thesis, University of St Andrews.

Cäsar Cristiane, Byrne Richard, Hoppitt William, Young Robert J. and Zuberbühler Klaus, 2012a. Evidence for semantic communication in Titi monkey alarm calls. Anim. Behav. 84: 405-411. doi:10.1016/j.anbehav.2012.05.010.

Cäsar Cristiane, Byrne Richard, Young Robert J. and Zuberbühler Klaus, 2012b. The alarm call system of wild black-fronted titi monkeys, Callicebus nigrifrons. Behav. Ecol. Sociobiol. 66(5): 653-667. doi:10.1007/s00265-011-1313-0.

Cäsar Cristiane, Zuberbühler Klaus, Young Richard J. and Byrne Richard, 2013. Titi monkey call sequences vary with predator location and type. Biol Lett 9: 20130535. doi:10.1098/rsbl.2013.0535.

Chierchia, Gennar; Fox, Danny; Spector, Benjamin. 2012. The Grammatical View of Scalar Implicatures and the Relationship between Semantics and Pragmatics. In Paul Portner, Claudia Maienborn and Klaus von Heusinger (eds), Handbook of Semantics, Vol. 3, 2297-2332. De Gruyter.

Collier Katie, Bickel Balthasar, van Schaik Carel P., Manser Marta B. and Townsend Simon W., 2014. Language evolution: syntax before phonology? Proceedings of the Royal Society of London, Series B: Biological Sciences 281: 1788. doi: 10.1098/rspb.2014.0263

Crockford, C., Wittig, R. M., Mundry, R. \& Zuberbuhler, K. 2012. Wild chimpanzees inform ignorant group members of danger. Current Biology, 22, 142e146.

Fedigan Linda M., 1990. Vertebrate Predation in Cebus capucinus: Meat Eating in a Neotropical Monkay. Folia Primatol. 54(3-4): 196-205. doi: 10.1159/000156444.

Grice Paul, 1975. Logic and conversation. In Syntax and Semantics, 3: Speech Acts, ed. P. Cole \& J. Morgan. New York: Academic Press.

Hattori Yuko, Kuroshima Hika, Fujita Kazuo, 2010. Tufted capuchin monkeys (Cebus apella) show understanding of human attentional states when requesting food held by a human. Animal Cognition 13: 87-92

Heim, Irene and Kratzer, Angelika. 1998. Semantics in Generative Grammar. Blackwell.

Horn Laurence R., 1972. On the semantic properties of the logical operators in English. Ph.D.dissertation, University of California at Los Angeles, Los Angeles, California.

Kaminski, Juliane; Call, Josep; Fischer, Julia, 2004. Word Learning in a Domestic Dog: Evidence for "Fast Mapping". Science 304 (5677): 1682-1683. doi:10.1126/science.1097859

Kershenbaum A., Bowles A.E., Freeberg T.M., Jin D.Z., Lameira A.R., Bohn K. , 2014. Animal vocal sequences: not the Markov chains we thought they were. Proceedings of the Royal Society $B$ 
Kershenbaum, A., Blumstein, D.T., Roch, M., et al. 2015. Acoustic sequences in non-human animals: a tutorial review and prospectus. Biological Reviews doi: 10.1111/brv.12160

Levinson, Stephen C. 2000. Presumptive Meanings: The Theory of Generalized Conversational Implicature. MIT Press

Papafragou, Anna \& Julien Musolino. 2003, Scalar implicatures: experiments at the semanticspragmatics interface. Cognition $86.253-282$.

Parrish A.E. and Brosnan S.F., 2012. Primate Cognition. In: V.S. Ramachandran (ed.) The Encyclopedia of Human Behavior, vol. 3, pp. 174-180. Academic Press.

Pepperberg, Irene M. 2010. Vocal learning in Grey parrots: A brief review of perception, production, and cross-species comparisons. Brain \& Language, 115, 81-91.

Schlenker Philippe: to appear, The Semantics/Pragmatics Interface. To appear in the Cambridge Handbook of Formal Semantics, eds. Aloni and Dekker.

Schlenker Philippe, Chemla Emmanuel, Arnold Kate, Lemasson Alban, Ouattara Karim, Keenan Sumir, Stephan Claudia, Ryder Robin and Zuberbühler Klaus: 2014, Monkey Semantics: Two 'Dialects' of Campbell's Monkey Alarm Calls. Linguistics \& Philosophy 37, 6: 439-501. DOI 10.1007/s10988-014-9155-7.

Schlenker, Philippe, Chemla, Emmanuel, Arnold, Kate, Zuberbühler, Klaus, to appear a, Pyow-hack Revisited: Two Analyses of Putty-nosed Monkey Alarm Calls. To appear in Lingua.

Schlenker, Philippe; Chemla, Emmanuel; Schel, Anne M.; Fuller, James; Gautier, Jean-Pierre; Kuhn, Jeremy; Veselinovic, Dunja; Arnold, Kate; Cäsar, Cristiane; Keenan, Sumir; Lemasson, Alban; Ouattara, Karim; Ryder, Robin; Zuberbühler, Klaus, to appear b. Formal Monkey Linguistics. Target article to appear in Theoretical Linguistics.

Seyfarth Robert M., Cheney Dorothy L and Marler Peter, 1980a. Monkey responses to three different alarm calls: evidence of predator classification and semantic communication. Science 210: 801803.

Seyfarth Robert M., Cheney Dorothy L. and Marler Peter, 1980b.Vervet monkey alarm calls: semantic communication in a free-ranging primate. Anim. Behav. 28: 1070-1094.

Shultz Susanne and Thomsett Simon, 2007. Interactions between African crowned eagles and their primate prey community. In: Monkeys of the Taï forest: an African monkey community. (eds McGraw WS, Zuberbühler K, Noë R) Cambridge Univ. Press, Cambridge, pp 171-193.

Wheeler Brandon C., Fischer Julia, 2012. Functionally referential signals: a promising paradigm whose time has passed. Evolutionary Anthropology 21: 195-205.

Zuberbühler Klaus, Jenny David and Bshary Redouan, 1999. The Predator Deterrence Function of Primate Alarm Calls. Ethology 105 (6): 477-490. doi: 10.1046/j.1439-0310.1999.00396.x.

Zuberbühler Klaus and Jenny David, 2007. Interactions between leopards and monkeys. In: Monkeys of the Taï Forest: An African monkey community. (eds McGraw WS, Zuberbühler K, Noë R) Cambridge Univ. Press, Cambridge.

Zuberbühler, Klaus: 2009, Survivor Signals: The Biology and Psychology of Animal Alarm Calling Advances in the Study of Behavior, Vol. 40. San Diego: Elsevier, pp. 277-322. 